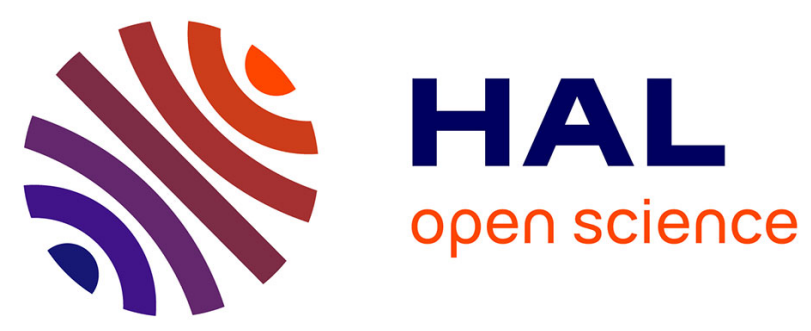

\title{
Étude par sondes électriques mobiles des différents paramètres d'un plasma de césium obtenu par ionisation de contact dans une machine à géométrie variable
} Marceau Felden, Claude Patou, Robert Haug

\section{- To cite this version: \\ Marceau Felden, Claude Patou, Robert Haug. Étude par sondes électriques mobiles des différents paramètres d'un plasma de césium obtenu par ionisation de contact dans une machine à géométrie variable. Revue de Physique Appliquée, 1966, 1 (1), pp.48-60. 10.1051/rphysap:019660010104800 . jpa-00242684}

\section{HAL Id: jpa-00242684 \\ https://hal.science/jpa-00242684}

Submitted on 1 Jan 1966

HAL is a multi-disciplinary open access archive for the deposit and dissemination of scientific research documents, whether they are published or not. The documents may come from teaching and research institutions in France or abroad, or from public or private research centers.
L'archive ouverte pluridisciplinaire HAL, est destinée au dépôt et à la diffusion de documents scientifiques de niveau recherche, publiés ou non, émanant des établissements d'enseignement et de recherche français ou étrangers, des laboratoires publics ou privés. 


\title{
ÉTUDE PAR SONDES ÉLECTRIQUES MOBILES DES DIFFÉRENTS PARAMÈTRES D'UN PLASMA DE CESIUM OBTENUIPAR IONISATION DE CONTACT DANS UNE MACHINE A GEOMÉTRIE VARIABLE
}

\author{
Par Marceau felden, Claude Patou et Robert HaUg, \\ Laboratoire de Physique Théorique et Nucléaire, Faculté des Sciences de Nancy.
}

\begin{abstract}
Résumé. - Lne machine à plasma de césium est étudiée. Elle est métallique et entièrement démontable ce qui permet de réaliser plusieurs structures différentes suivant les problèmes envisagés. Dans ce travail, la géométrie est variable et deux sondes mobiles analysent le milieu crée par ionisation de contact. Les mesures sont faites à différentes pressions pour des températures de surface chaude variables. Les électrodes, dont la polarisation peut être modifiée, sont placées à deux distances différentes. La première partie est consacrée à la description de la machine. Dans la seconde partie les résultats de plusieurs expériences sont présentés et discutés.
\end{abstract}

\begin{abstract}
A caesium plasma machine is studied. It is made of metal and is entirely demountable which allows different structures to be used for various problems. In this work, the geometry is variable and two moving probes analyse the region created by contact ionization. The measurements are made at different pressures for variable hot surface temperatures. Electrodes of which the polarization may be changed, are situated at two different distances. The first part describes the machine. In the second part, results of several experiments are presented and discussed.
\end{abstract}

Introduction. - Une vapeur métallique peut venIr s'ioniser au contact d'une surface chaude si certaines conditions physiques sont remplies. Ce phénomène, observé pour la première fois par Langmuir [1], a été expliqué par la thermodynamique. Si on considère un gaz monoatomique en équilibre, on obtient la loi de Saha-Langmuir $[2,3]$ qui s'écrit

$$
n_{\boldsymbol{i}}^{\prime} \times n_{\boldsymbol{e}}^{\prime} / n_{0}=\left(2 \pi m k T / h^{2}\right)^{3 / 2} \exp \left(-e V_{\mathbf{i}} / k T\right) .
$$

Dans cette relation $n_{i}^{\prime}$ et $n_{e}^{\prime}$ sont les densités ioniques et électroniques au voisinage immédiat de la surface chaude et $n_{0}$ représente la densité des particules neutres. Les autres paramètres sont les suivants : $m$ représente la masse de l'électron, $k$ la constante de Boltzmann, $h$ la constante de Planck, T la température d'équilibre (supposée égale à la température de la surface chaude $T_{\mathbf{E}}$ ) et $V_{\mathbf{i}}$ le potentiel d'ionisation des atomes de la vapeur métallique.

Par ailleurs une surface métallique chaude émet des électrons selon un processus qui est régi par l'équation de Richardson $[4,5,6]$ :

$$
n_{\mathbf{e}}^{\prime}=2\left(2 \pi m k T / h^{2}\right)^{3 / 2} \exp \left(-e \varphi_{\mathbf{E}} / k T_{\mathbf{E}}\right)
$$

dans laquelle $\varphi_{E}$ représente le potentiel de sortie des électrons pour la surface métallique considérée à la température $T_{\mathrm{E}}$.

Compte tenu de ces deux équations, le rapport de la densité ionique $n_{\boldsymbol{i}}^{\prime}$ à la densité d'atomes neutres $n_{0}$ est donné par la relation :

$$
n_{\boldsymbol{i}}^{\prime} / n_{\mathbf{0}}=(1 / 2) \exp \left(e\left(\varphi_{\mathbf{E}}-V_{\mathbf{i}}\right) / k T_{\mathbf{E}}\right) .
$$

Selon les conditions expérimentales, on peut obtenir soit un excès d'ions soit un excès d'électrons devant la surface d'émission ce qui crée une zone à prédominance de charges positives ou négatives suivant les cas.

Dans une telle région, appelée gaine, la densité des porteurs de charges $\left(n_{i}^{\prime}\right.$ et $\left.n_{e}^{\prime}\right)$ est généralement différente de celle du milieu ( $n_{\mathbf{i}}$ et $\left.n_{\mathrm{e}}\right)$.

La nature et la température de la surface métallique chaude déterminent complètement l'émission électronique mais elles ne conditionnent que partiellement la densité ionique puisque celle-ci dépend également de la pression de vapeur de l'élément à ioniser. La modification de ces différents paramètres permet done de faire varier les propriétés du milieu ionisé.

Description de la machine. - Nous avons utilisé les résultats précédents pour réaliser une machine à plasma de grand volume (250 centimètres cubes). Cette machine est entièrement démontable ce qui nous permet de la faire fonctionner dans des conditions très différentes : diode ou structure plus ou moins complexe possédant des électrodes (grilles) de configuration variable. 
Nous allons examiner des résultats qui ont été obtenus avec un montage en diode à collecteur mobile pendant les expériences, mais d'autres travaux ont été réalisés avec des configurations différentes. Nous avons utilisé de la vapeur de césium pour créer le milieu ionisé.

Principaux paramètres. - Pression de CÉsium. - La pression de césium est déterminée par la température $T_{\text {cs }}$ d'un réservoir contenant ce métal si ce réservoir est le point le plus froid de l'installation. Dans ce cas, pour $280^{\circ} \mathrm{K}<T_{\text {cs }}<1000$ oK, la pression d'équilibre est donnée par la relation $[7,8]$ : ${ }_{\alpha} \log P_{\text {es }}($ torr $)=11,38$

$$
\text { - 1,45 Log } T_{\text {cs }}-\left(4075 / T_{\text {cs }}\right) \text {. }
$$

Il faut donc réaliser le meilleur vide possible dans une enceinte dont les parois sont portées à une température supérieure à celle du réservoir de césium.

$\mathrm{E}_{\mathrm{n}}$ général la température de la machine doit être assez élevée puisque pour $T_{\mathrm{cs}}=450^{\circ} \mathrm{K}$ la pression est inférieure à $10^{-1}$ torr. Pour des raisons technologiques nous nous sommes fixé cette valeur comme limite supérieure puisqu'elle exige de porter en permanence l'ensemble de l'installation à une température de l'ordre de $500^{\circ} \mathrm{K}$ ce qui pose des problèmes en raison de la grande réactivité chimique du césium.

Une ampoule de verre scellée, contenant quelques grammes de césium (marque KAWECKI pureté $99,9 \%$ ) est placée dans le réservoir avant toute expérience. Cette ampoule est brisée après l'étuvage au moyen d'une masselotte métallique actionnée par un électroaimant externe.

La mesure directe de pression est délicate. Nous avons essayé plusieurs types de jauges dont une qui fonctionnait sur le principe de l'ionisation de contact mais certains problèmes technologiques restent à résoudre. Pour éviter ces difficultés, on utilise la relation ci-dessus en fixant la température du césium métallique qui est toujours le point le plus froid de l'enceinte. Des corrections imposées par les dimensions des canalisations $[9,10]$, ont été faites et on a vérifié le bon accord, aux imprécisions expérimentales près, entre la valeur de la pression de césium calculée à partir de la seule connaissance de la température $T_{\text {cs }}$ du point froid et la valeur de cette même pression mesurée par l'émission électronique d'une jauge constituée d'un filament entouré par un collecteur cylindrique muni d'anneaux de garde. Dans tous les cas plusieurs heures sont nécessaires pour que l'équilibre s'établisse, ce qui est observé par l'évolution des caractéristiques électriques de la machine.

Température de la surface chaude. - Les travaux de Langmuir et de Nottingham [11, 12] ont montré que la température $T_{\mathbf{E}}$ de la surface chaude, ou émetteur, doit être supérieure ou égale à une température minimale $T_{\mathrm{E}_{0}}$ pour que tout atome de césium qui vient à son contact soit ionisé ; ce seuil dépend de la pression de césium donc de la température du point froid.

Comme la limite supérieure de température du point froid est égale à $500^{\circ} \mathrm{K}$, on montre [13] que $T_{\mathrm{E}_{0}}=1800^{\circ} \mathrm{K}$. Notre machine nous permettant de porter la surface chaude à une température supérieure à $2200^{\circ} \mathrm{K}$, nous sommes donc assurés, en choisissant convenablement nos paramètres, de pouvoir ioniser tous les atomes de césium incidents.

La surface métallique chaude émet des électrons dont le spectre de vitesse est sensiblement maxwellien dès leur sortie du métal [14]. (Rappelons que leur distribution de vitesses est soumise à la statistique de Fermi-Dirac à l'intérieur du métal [15].) La présence éventuelle de vapeur de césium sur l'émetteur modifie son travail de sortie et la loi d'émission électronique est plus compliquée que celle de Richardson ; elle est donnée par les courbes caractéristiques de Langmuir $[11,16]$. Dans tous les cas ces électrons peuvent entrer en interactions avec la vapeur métallique, ionisée ou non, la probabilité de ces interactions dépendant des conditions physiques. Ces phénomènes ont servi d'éléments de base dans la construction de notre machine puisque des travaux antérieurs $[17,18,19]$ indiquent que : pour $T_{\text {cs }}=350$ oK on a $P_{\mathrm{cs}}=10^{-4}$ torr et $\lambda_{\mathrm{e}}=10^{\mathrm{cm}}$ et pour $T_{\mathrm{cs}}=500^{\circ} \mathrm{K}$ alors $P_{\mathrm{cs}}=2 \times 10^{-1}$ torr et $\lambda_{e}=2 \times 10^{-3} \mathrm{~cm}$ où $\lambda_{e}$ est le libre parcours moyen de choc élastique pour des électrons d'énergie $1 \mathrm{eV}$. A l'aide de ces chiffres et de ceux qui sont cités en référence on peut calculer que si la machine permet aux électrons de parcourir la distance $d$, deux cas limites sont à envisager :

a) $d \ll \lambda_{\mathrm{e}}$ il n'y a pratiquement pas d'interactions et on est sensiblement en flux de particules libres ;

b) $d \gg \lambda_{\mathbf{e}}$ il y a de grandes probabilités d'interactions et on est en régime collisionnel.

C'est la raison pour laquelle, compte tenu des limites de pression de vapeur de césium imposée par la technologie, notre machine a été construite pour que :

$$
10^{-3}<\lambda_{\mathrm{e}} / d<10^{2}
$$

Dans ce cas la distance interélectrodes $d$ varie entre pratiquemment zéro et dix centimètres. La loi de Saha montre que, dans nos conditions expérimentales, une gaine ionique se crée devant l'émetteur, de plus on connaît la répartition théorique du potentiel dans cette gaine [3], [9].

Remarquons encore qu'il existe de nombreux processus d'interactions entre le faisceau d'électrons et la vapeur métallique mais avant toute étude il faut, au préalable, mettre au point des méthodes précises de mesure des paramètres du plasma, c'est un des objectifs de ce travail. 
Réalisation de la machine. - Nous avons construit une machine de grand volume pouvant fonctionner à une température de l'ordre de 500 o $\mathrm{K}$ pendant plusieurs jours. Ceci nécessite un étuvage préalable de plusieurs heures à une température voisine de $650^{\circ} \mathrm{K}$ pour que le vide statique reste bon pendant la durée de l'expérience. Le choix des matériaux est donc très important aussi bien pour leur tenue au vide à haute température que pour leur comportement en présence de vapeur de césium $[20,21]$. Certains résultats antérieurs $[10,22,23]$ donnent des renseignements sur ce sujet. En particulier, la présence de césium exclut l'emploi de silice donc de matériaux tels que le quartz, le verre, etc...

Description générale. - La figure 1 représente une vue générale de la machine et de son groupe de

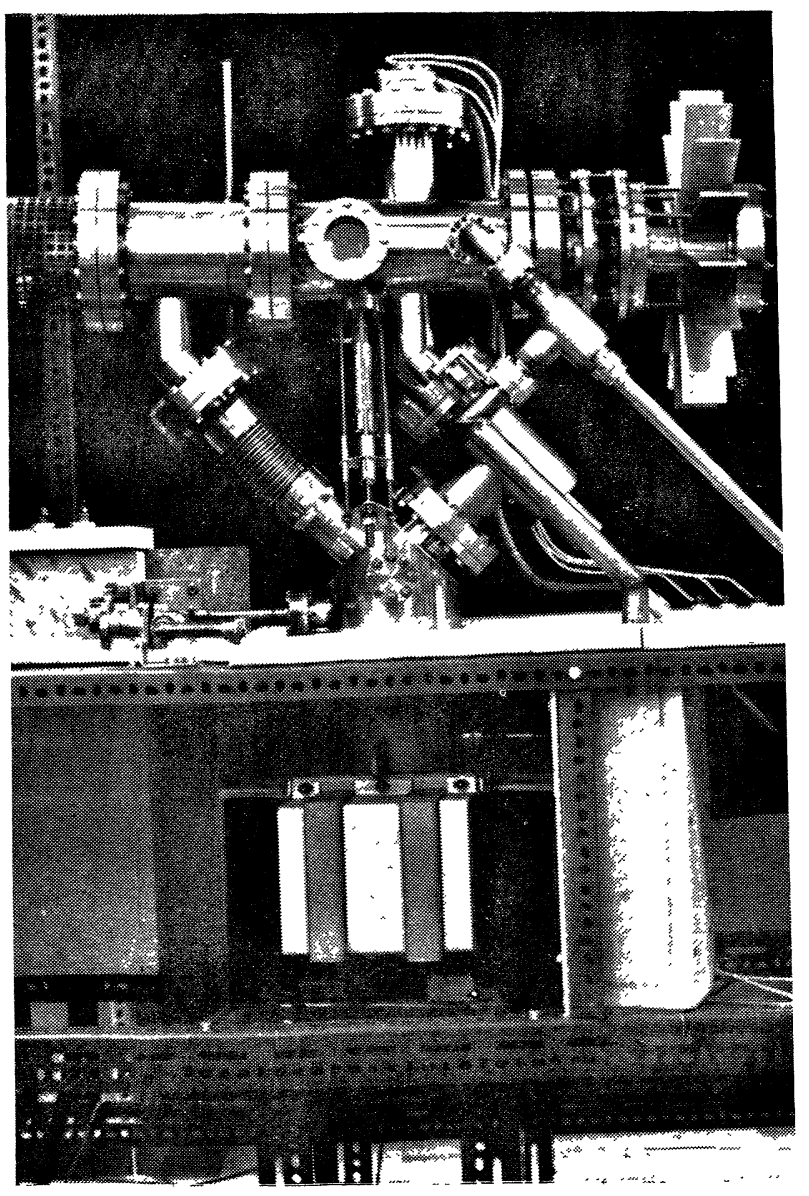

Fig. 1.

pompage (pompe ionique de marque ULTEK, débit 200 litres par seconde). Elle est réalisée en acier inoxydable non magnétique (type NS $22 \mathrm{~S}$ ). Le détail est représenté sur la figure 2 . La partie principale de l'appareil est constituée par un cylindre de

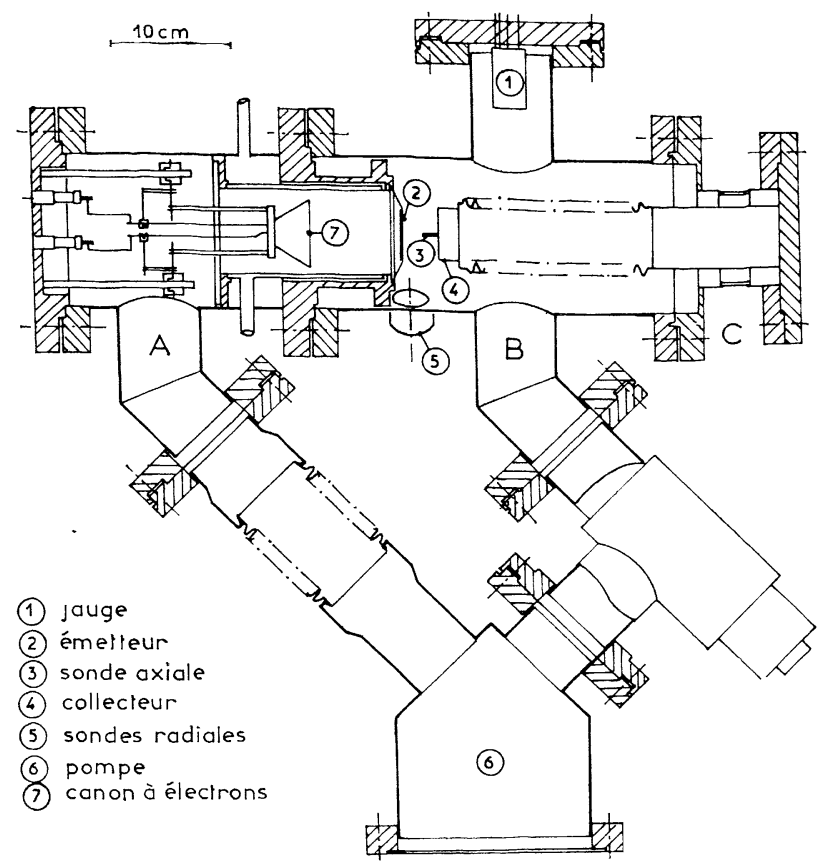

FIG. 2.

$600 \mathrm{~mm}$ de long et de $120 \mathrm{~mm}$ de diamètre intérieur qui est divisé en trois éléments que nous allons examiner.

La partie A (cylindre entre les deux brides à gauche sur la figure 1) contient le canon à électrons destiné à chauffer la surface chaude qui ferme de manière étanche sa face droite ( $f g .2)$. Ce montage étanche isole cette section du reste de l'installation pour éviter la présence de vapeur de césium dans le canon. L'autre extrémité est fermée par une bride munie de passages de courant. Une canalisation de $60 \mathrm{~mm}$ de diamètre intérieur relie en permanence cette section au groupe de pompage.

L'émetteur est en métal réfractaire à potentiel de sortie $\varphi_{E}$ élevé. Le molybdène, le tungstène et le tantale ont été essayés et c'est ce dernier métal qui a le mieux résisté aux cycles thermiques tout en restant imperméable à la vapeur de césium, condition impérative au bon fonctionnement du canon. La surface chaude a la forme d'une cuvette de

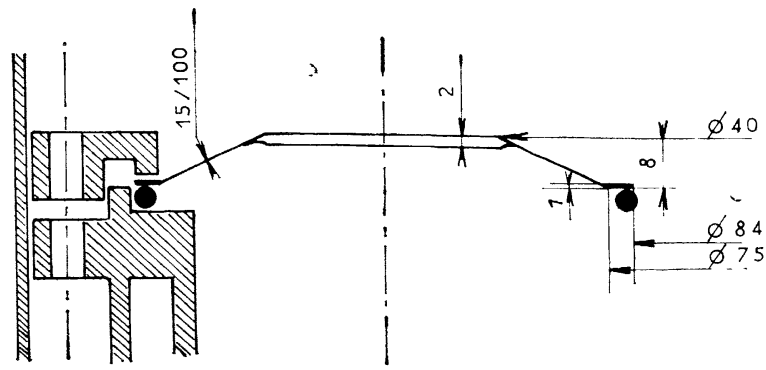

F IG. 3 . 


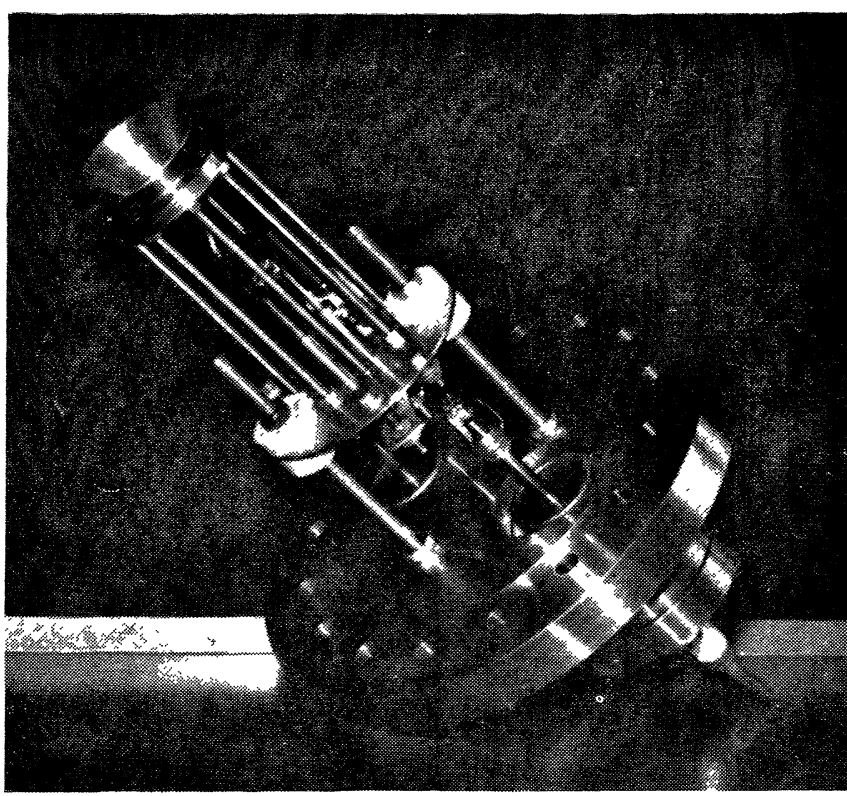

FIG. 4.

84 $\mathrm{mm}$ de diamètre qui possède une épaisseur de $2 \mathrm{~mm}$ sur la partie chauffée et de 15/100 de mm sur la partie évasée (fig. 3). Cette structure limite la conduction thermique. Le joint d'étanchéité interne est en platine, il est refroidi par circulation d'air dans une double paroi visible sur la figure 2 . Un thermocouple contrôle en permanence sa température. La surface d'émission est géométriquement bien définie par la présence, devant l'émetteur, d'une couronne en tantale de $40 \mathrm{~mm}$ de diamètre intérieur. Cette couronne est au potentiel de l'émetteur, elle sert d'anneau de garde.

Le canon à électrons est représenté, sur la figure 4. Il est constitué par un filament spiralé en fil de tungstène de $0,5 \mathrm{~mm}$ de diamètre qui est chauffé par effet Joule. Derrière se trouve un réflecteur en acier inoxydable porté au même potentiel. La forme de ce réflecteur et la géométrie du canon ont été étudiées expérimentalement pour obtenir un chauffage homogène de l'émetteur sur une surface circulaire de $40 \mathrm{~mm}$ de diamètre. On y parvient avec un gradient de $50^{\circ} \mathrm{K}$ environ entre le bord et le centre pour une température de $2000{ }^{\circ} \mathrm{K}$. La mesure est faite par pyrométrie optique compte tenu des facteurs de corrections [24]. Ce canon est relié à une alimentation qui permet de fournir 10 kilovolts et 500 milliampères. Le canon travaille en régime de charge d'espace et sa pervéance permet à l'alimentation de donner son maximum de puissance.

Le détail et les sécurités de l'alimentation sont représentées sur la figure 5. Elle fonctionne à courant constant contrôlé par réglage du courant continu parcourant des enroulements d'inductances à saturation variable, placées au primaire du transformateur principal. Ce dispositif permet une sécurité relative pendant l'étuvage du canon. En cas de remontée de pression supérieure à $10^{-4}$ torr un disjoncteur arrête l'alimentation de la pompe ionique et du canon à électrons. Un relais à minimum de courant agit de même en cas de rupture du filament.

La partie B (cylindre central) contient le volume utile proprement dit dans lequel est créé le milieu ionisé. Cette section comporte un certain nombre d'orifices munis de brides de raccord. Sur la partie inférieure se trouve une canalisation de $60 \mathrm{~mm}$ de diamètre qui est reliée à la pompe par l'intermédiaire d'une vanne $V_{1}$ (Vacuum Generatrors de $75 \mathrm{~mm}$ d'ouverture) étuvable en position ouverte ou fermée à $600^{\circ} \mathrm{K}$. Elle peut être manœuvrée à cette température, grâce à un système prolongateur externe. Cette vanne permet d'isoler le volume utile du groupe de pompage avant l'injection de vapeur de césium qui provient du métal contenu dans le réservoir D muni d'un four autonome (visible sur la figure 1) régulé à $\pm 1{ }^{\circ} \mathrm{C}$ près. La vapeur est envoyée dans la partie $B$ à travers la vanne $V_{1}$ (VARIAN ouverture 43,2 $\mathrm{mm}$ ), par une canalisation de $30 \mathrm{~mm}$ de diamètre intérieur. Cette section possède également un tube supérieur avec bride de raccord qui permet de monter une jauge à vide ou tout autre système électrique nécessaire aux expériences. Il y a deux hublots en saphir (CSF, $50 \mathrm{~mm}$ de diamètre) qui servent à toutes les visées optiques nécessaires (mesure de température de l'émetteur, distance émetteur collecteur, examen des sondes, de leur position, étude du milieu, etc...) et éventuellement au passage d'un faisceau U. H. F. Enfin trois orifices disposés de manière dissymétrique par rapport à l'axe du cylindre permettent l'introduction de sondes mobiles munies de leur mécanisme de translation (on peut en voir une sur la figure 1 en dessous du hublot). Ils servent également à l'introduction éventuelle de diverses informations électriques dans le volume utile (par exemple : polarisation d'électrodes diverses).

La partie C est variable suivant les expériences envisagées. Dans le cas présent elle est constituée par un collecteur mobile en cuivre de $40 \mathrm{~mm}$ de diamètre qui se déplace sur une distance de $50 \mathrm{~mm}$ devant la surface chaude. Il peut théoriquement venir à son contact, seule la présence d'une sonde radiale mobile proche de l'émetteur limite cette possibilité. Le déplacement est mesuré au cathétomètre à travers les hublots. Le collecteur est isolé électriquement de l'enceinte par un anneau en céramique de $70 \mathrm{~mm}$ de diamètre (C. F. T. H.). Il possède également une sonde axiale mobile. Les ailettes que l'on distingue sur la figure 1 servent à son refroidissement par conduction thermique. Un thermocouple proche de sa surface, montre qu'il reste à une température inférieure à $600^{\circ} \mathrm{K}$ ce qui permet son recouvrement en césium. 


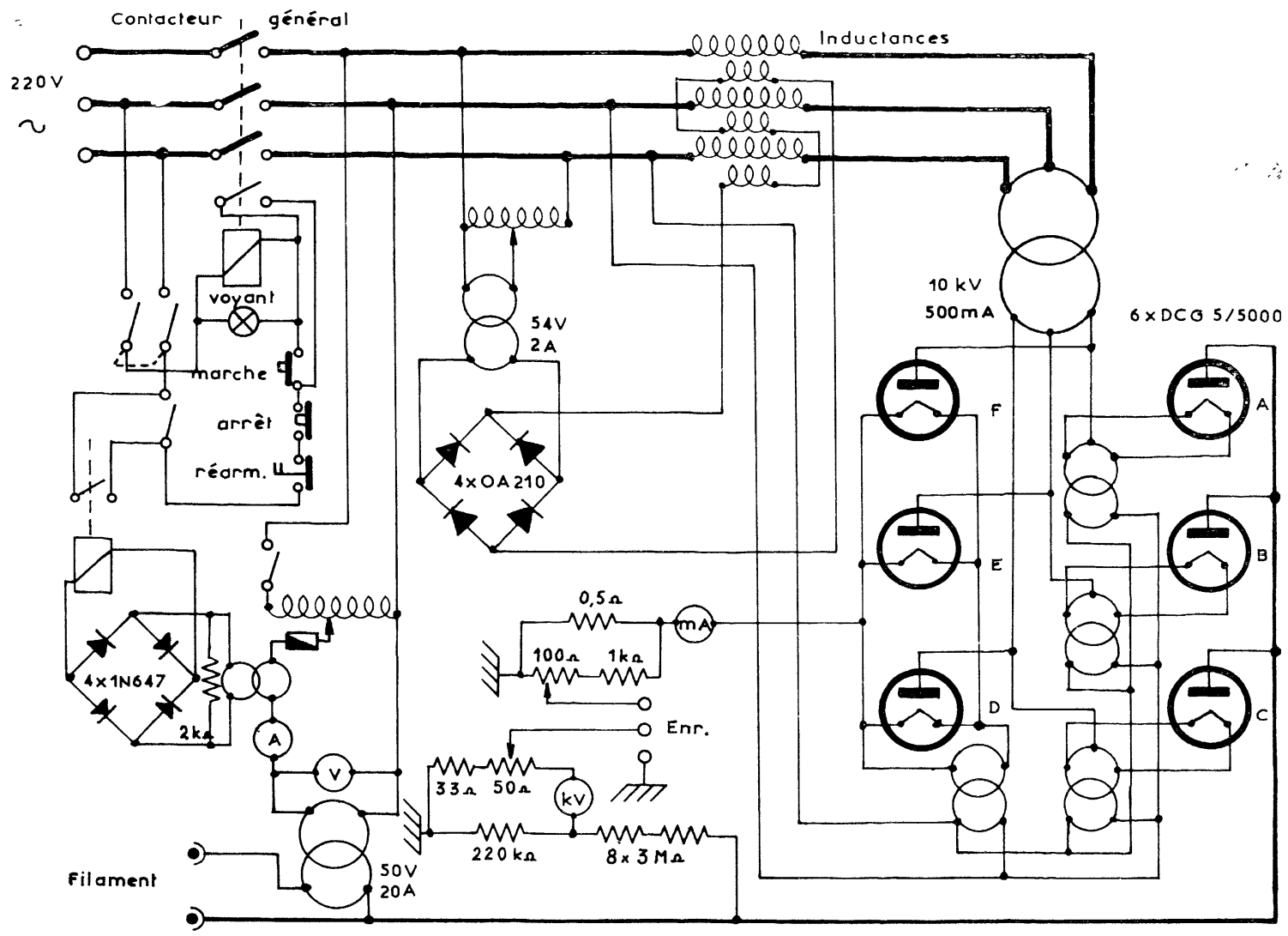

FIg. 5. - Schéma de l'alimentation du canon à électrons.

Les joints sont en fil d'or de $0,5 \mathrm{~mm}$ de diamètre pour les parties de l'enceinte qui ne sont pas en contact avec la vapeur de césium, ils sont en platine pour les autres. La figure 6 représente une coupe des brides de raccord. Chaque joint est placé au

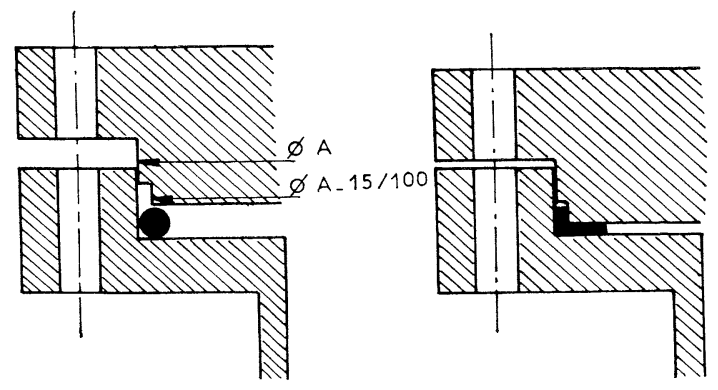

FIG. 6.

fond d'une gorge de $2 \mathrm{~mm}$ de profondeur et la pièce mâle comporte une partie émincée de 15/100 de mm permettant au joint de prendre une forme de L. Cette structure résiste bien aux variations thermiques imposées par les cycles d'étuvages et par les diverses expériences envisagées.
Nous utilisons, dans la mesure du possible, une qualité d'alumine avec un taux d'impuretés en silice inférieur à 10-4 (LUCALOX General Electric) pour éviter l'attaque chimique due au césium.

Cycle expérimental. - Au début de toute expérience, un four est descendu sur la machine. Il est muni de deux galets en pyrex face aux hublots. Le chauffage est obtenu par circulation forcée d'air passant sur plusieurs groupes de résistances électriques dont l'alimentation est contrôlée et régulée selon un montage indiqué sur la figure 7 . Un thermocouple Th mesure la température de l'air. Si la mesure est inférieure à la température imposée, un moteur électrique M solidaire du curseur d'un auto-transformateur (circuit 1), augmente la tension appliquée aux résistances $R_{1}$ et le contact se ferme pendant un intervalle de temps prérég]é. Les trois résistances $R_{2}$ apportent alors une puissance thermique supplémentaire. A pleine puissance, les circuits 1 et 2 dissipent respectivement 6 et $1 \mathrm{~kW}$. Si la mesure du thermocouple est supérieure à la valeur imposée, le moteur $\mathrm{M}$ agit sur le transformateur pour diminuer la tension appliquée au circuit 1. 


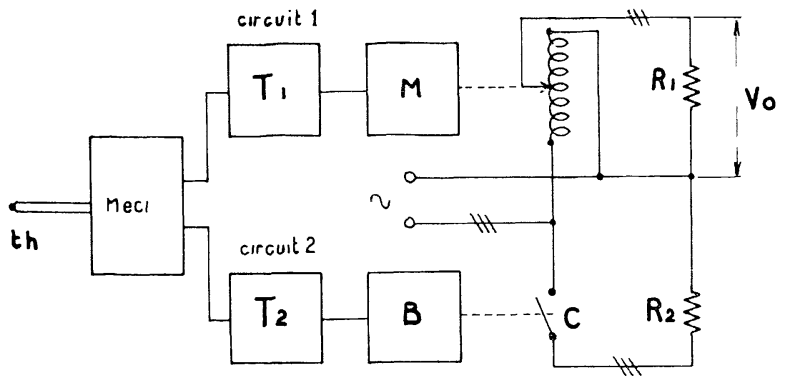

FIg. 7. - Régulation de la température du four.

Le dégazage est effectué pendant environ 1.8 heures à une température de l'ordre de 600 à $650^{\circ} \mathrm{K}$. Ensuite elle est ramenée à la valeur imposée par le cycle expérimental compte tenu du fait que l'enceinte doit rester à une température supérieure à celle du réservoir contenant le césium.

L'expérience montre que, après l'étuvage, la pression résiduelle n'excède pas $5 \times 10^{-8}$ torr à une température de $450^{\circ} \mathrm{K}$. A la température ambiante elle est voisine de $4 \times 10^{-10}$ torr.

Pendant tout cycle expérimental qui peut s'étendre de manière continue pendant plusieurs jours, de nombreuses informations sont enregistrées en permanence pour assurer le contrôle de la machine.

Sondes électriques. - La théorie des sondes de Langmuir est bien connue $[25,26,27,28]$ mais leur utilisation pose quelques problèmes surtout en présence de vapeur de césium. L'électrode de collection plane est réalisée en fil de tungstène de $0,5 \mathrm{~mm}$ de diamètre. Il est supérieur à la distance de Debye [27]. Chaque sonde possède un anneau de garde constitué par un tube en acier inoxydable de 1,4 mm de diamètre intérieur et $1,8 \mathrm{~mm}$ de diamètre extérieur. La figure 8 représente les divers éléments d'une sonde : la partie (1) est une vue d'ensemble

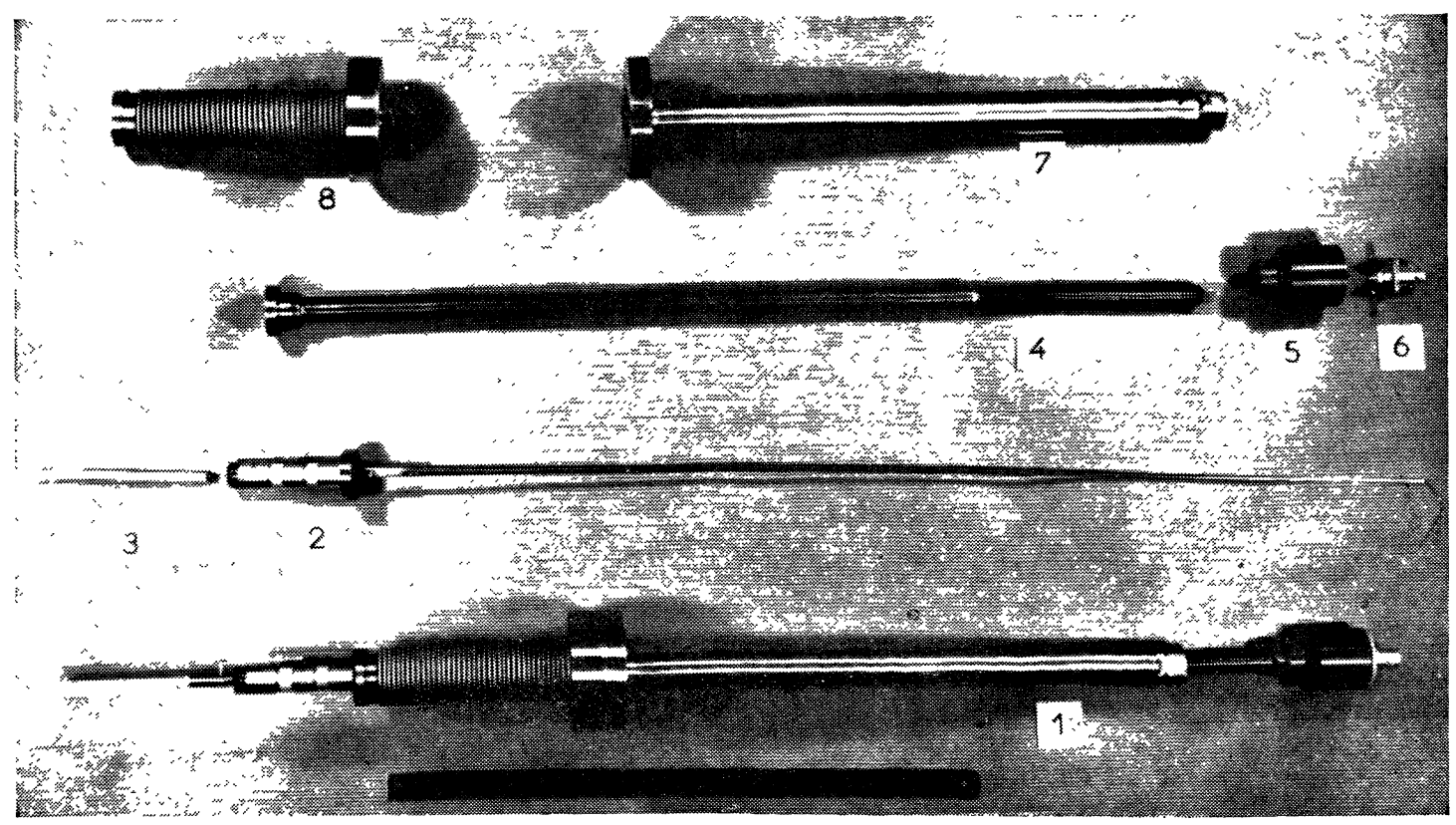

FIG. 8.

avec les différents mécanismes de translation et la bride de raccord.

La sonde elle-même est représentée par la partie (2) avec son extrémité (3) qui est placée dans le milieu ionisé. Cette section comprend une âme centrale métallique (électrode de collection) gainée par un tube d'alumine lui-même placé dans un tube en acier inoxydable servant d'anneau de garde, enfin un tube extérieur en alumine protège le tout. On remarque la section à double isolement à l'extré- mité gauche de (2). Les éléments (4) et (7) représentent le mécanisme de translation externe comportant la membrane souple (8). Enfin les parties.(5) et (6) représentent le dispositif d'isolement électrique et de raccord aux circuits extérieurs. Deux blindages permettent d'être assuré que seule l'extrémité de la sonde collecte les charges.

Un point délicat concerne la mesure de la température de la surface de collection de la sonde qui conditionne son recouvrement en césium donc son 
travail de sortie $\varphi_{\text {s. }}$ La mesure du potentiel local nécessite la connaissance de ce paramètre. Comme la température varie en fonction de la position de la sonde et de la température de la surface chaude, on se heurte à des difficultés expérimentales qui ont été levées en faisant un étalonnage préalable dans lequel l'électrode de collection a été remplacée par un thermocouple. Dans des expériences récentes, qui ne sont pas décrites ici, nous avons utilisé des sondes refroidies.

Les résultats que nous allons examiner ont été obtenus avec deux sondes. L'une est radiale, elle peut se déplacer à $8 \mathrm{~mm}$ de l'émetteur, parallèlement à son plan, entre le centre et le bord. Nous la désignerons S. R. (sonde radiale). L'autre sonde, mise au centre du collecteur, peut se déplacer entre $-10 \mathrm{~mm}$ et $+40 \mathrm{~mm}$ par rapport à sa face terminale. Elle est mobile le long de l'axe du cylindre, nous l'appelons sonde axiale $\mathrm{S}$. A. Un dispositif d'enregistrement donne la courbe $I=f(V)$ et les dérivées première et seconde [29].

Résultats expérimentaux. - La répartition du potentiel dans l'espace interélectrodes dépend de la différence de potentiel $V_{\mathrm{CE}}$, en général constante appliquée entre le collecteur et l'émetteur, la référence étant le niveau de Fermi de la surface chaude. Quand la distance interélectrode est grande, les parois peuvent jouer un rôle important en drainant un courant électronique latéral non négligeable.

Dans les résultats qui sont examinés, la sonde axiale est placée à $16 \mathrm{~mm}$ (notée SA 16) ou à $22 \mathrm{~mm}$ (notée SA 22) devant le collecteur. La sonde radiale est placée soit au voisinage immédiat de l'axe de la machine soit à environ $20 \mathrm{~mm}$ de cet axe ce qui correspond à la périphérie de l'émetteur.

Nous allons principalement étudier la densité électronique (exprimée en nombre d'électrons par centimètre cube) en fonction de la température d'émetteur pour différentes conditions physiques. Chaque réseau de courbes ne dépend en général que d'un seul paramètre. Nous nous sommes efforcés de grouper nos résultats par séries de mesures effectuées au cours du même cycle pendant lequel la machine n'est pas remise à l'air. Cette précaution est nécessitée par la difficulté de reproduire exactement les mêmes conditions expérimentales surtout en ce qui concerne la pression de la vapeur de césium, il est donc délicat de comparer complètement des résultats provenant de cycles différents. Ceci explique qu'il peut manquer certaines informations dans une série de mesures.

Étude de L'influence de LA PRession de CÉSIUM. - La pression de césium est le paramètre le plus imprécis. Des phénomènes de surface complexes tels que l'adsorption, la désorption et la formation d'oxydes de césium sont toujours à craindre. De ce fait la reproductibilité entre plusieurs cycles d'expériences est mauvaise. Tous nos résultats sont donnés à partir d'une même série expérimentale (machine non remise à l'air) ce qui fait qu'ils sont satisfaisants en valeur relative.

"Dans ces expériences, la distance émetteurcollecteur est de $43 \mathrm{~mm}$ et la différence de potentiel $V_{\mathrm{CE}}$ est de -4 volts.

Mesures en sonde axiale. - Sur la figure 9 nous avons représentéles variations de densité électro-

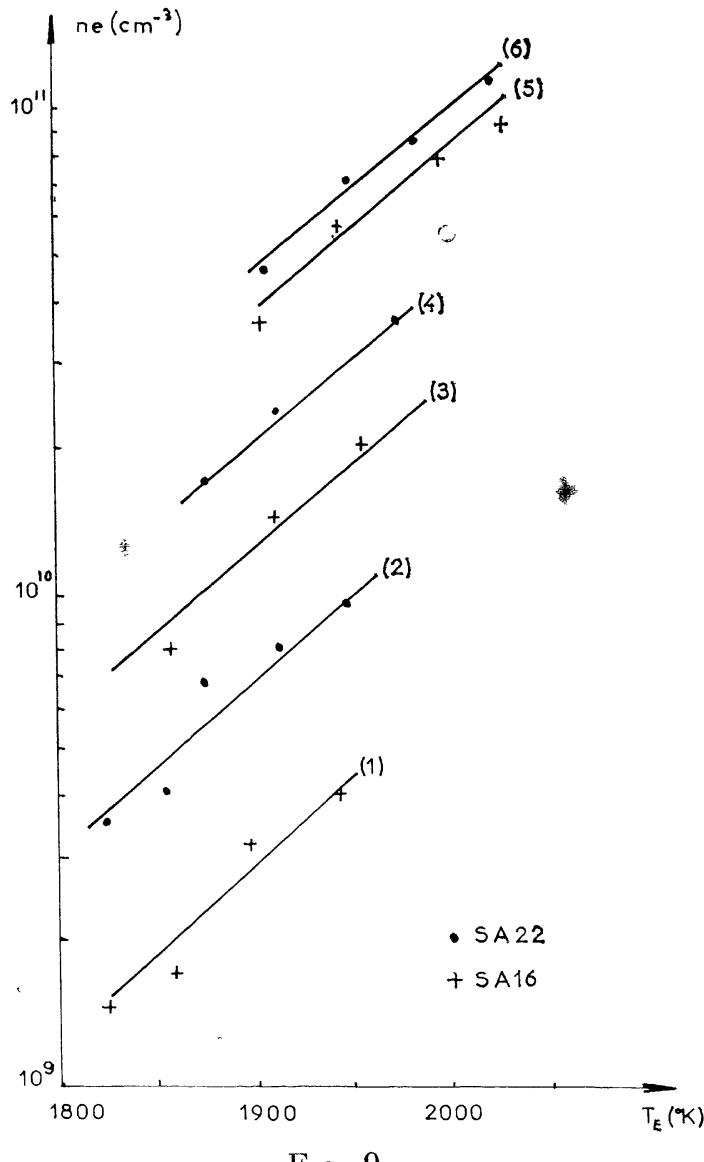

FIG. 9.

nique mesurées par la sonde axiale en fonction de la température de l'émetteur pour différentes pressions de césium. Toutes les mesures sont obtenues pour deux positions différentes de la sonde axiale : dans la première, la surface de collection est placée à $16 \mathrm{~mm}$ de la surface du collecteur (SA 16) dans la seconde, elle est placée à $22 \mathrm{~mm}$ (SA 22). Dans tous les cas on peut observer que la densité électronique mesurée sur l'axe croît quand on se rapproche de l'émetteur ce qui peut être lié à une diffusion quand la distance émetteur collecteur est grande. Les positions de la sonde axiale ont été choisies pour éviter de se placer dans les gaines.

Les courbes 1 et 2 correspondent à une pression 
de césium égale à $10^{-6}$ torr et nous constatons que la densité mesurée dans les mêmes conditions physiques dépend de la position de la sonde axiale. Le rapport moyen des mesures de densité en fonction de la position est proche de 2,5 . Les courbes 3 et 4 correspondent à une pression de césium de $5 \times 10^{-4}$ torr. Dans ce cas les densités mesurées dépendent moins fortement de la position puisque leur rapport moyen est voisin de 1,6. C'est encore le cas pour les courbes 5 et 6 qui correspondent à une pression de césium de $10^{-2}$ torr, le rapport moyen des mesures de densité est proche de 1,2. Il semble donc que la cohérence des mesures croît avec la pression de césium. Les variations relatives de densité en fonction de la température de l'émetteur semblent dépendre assez peu de la position de la sonde axiale à pression donnée ce qui montre qu'il n'y a pas de processus de saturation dans nos conditions expérimentales.

Par ailleurs, les densités mesurées à deux distances différentes se rapprochent de la même valeur quand la pression de césium croît puisque le rapport moyen des mesures de densité passe de 2,5 à 1,2 quand la pression augmente de $10^{-6}$ à $10^{-2}$ torr. On peut remarquer que les mesures correspondent à des températures d'émetteur de plus en plus élevées quand la pression de césium croît. En effet, compte tenu de l'augmentation du courant ionique en fonction de la pression, pour maintenir la neutralité, il faut faire croître la température de l'émetteur. Les mesures à forte pression et faible température d'émetteur deviennent inexploitables.

Mesures en sonde radiale. - Dans les mêmes conditions que précédemment, nous avons fait des mesures en sonde radiale en position centrale (SRC), et en position périphérique, c'est-à-dire à $20 \mathrm{~mm}$ de l'axe (SRP). Les résultats sont représentés sur la figure 10. Les courbes (1) et (2) correspondent encore à une pression de césium de $5 \times 10^{-4}$ torr alors que les courbes (3) et (4) sont relatives à une pression de césium de $10^{-2}$ torr.

Lorsque la sonde radiale est proche de l'axe, nous obtenons les courbes (2) et (4) qui confirment les résultats des courbes de la figure 10, en particulier les densités électroniques mesurées sont plus grandes à pression équivalente puisque cette sonde est plus proche de l'émetteur $(8 \mathrm{~mm})$.

Les pentes sont légèrement supérieures, c'est-àdire que la densité varie plus fortement en fonction de $T_{\mathrm{E}}$. La précision n'est cependant pas suffisante pour conclure. Les courbes (1) et (3) sont obtenues à partir de la sonde en position périphérique et on constate que les densités mesurées sont nettement plus faibles ainsi que la pente. Pour interpréter ces résultats il faut tenir compte du gradient de température entre le centre et la périphérie de l'émetteur, la diffusion des particules pouvant également intervenir. On peut cependant remarquer que dans les

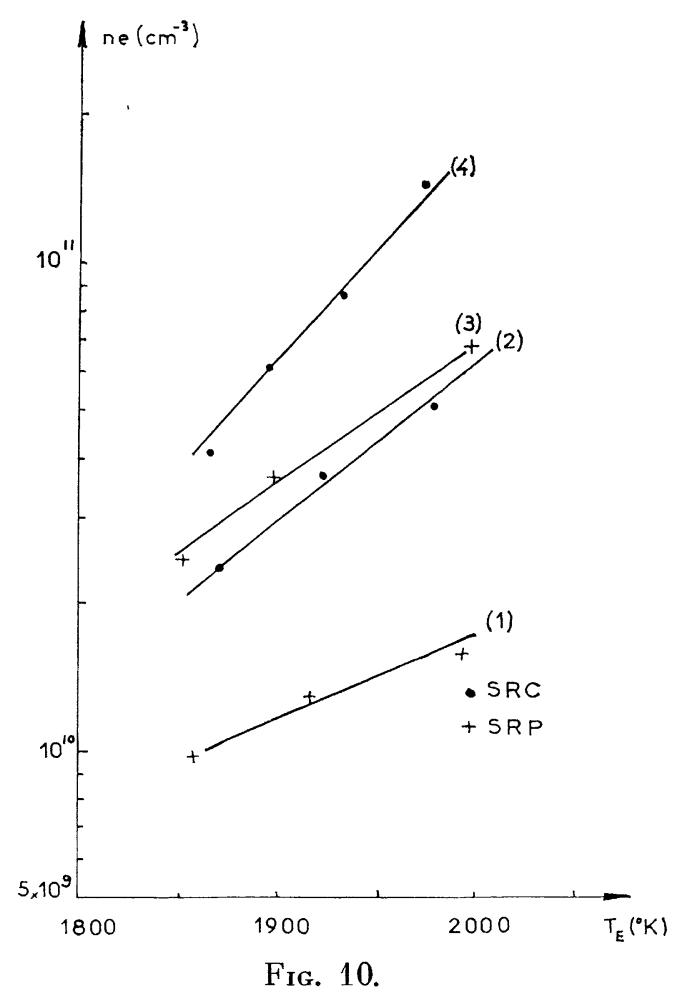

mêmes conditions expérimentales, le rapport moyen des densités mesurées par la sonde radiale placée au centre ou en position périphérique est voisin de 2,2 (estimation faite de $1950 \mathrm{o}$ K), quand la pression est de $10^{-2}$ torr alors qu'il est voisin de 3 pour une pression de $5 \times 10^{-4}$ torr. Il semble donc que les mesures se rapprochent quand la pression de césium croît.

Influence de la distance interélectrodes. - Nous avons déjà indiqué que la distance émetteur collecteur $d$ peut varier entre 0 et $50 \mathrm{~mm}$, cependant la présence des sondes ne permet pas de choisir toutes les positions possibles. Pour des raisons pratiques nous avons essentiellement travaillé à deux distances qui sont respectivement de 43 et $31 \mathrm{~mm}$.

Nous avons observé que la densité dépend de ce paramètre, mais il faut également considérer la pression de césium et de la différence de potentiel collecteur émetteur.

La figure 11 représente les variations de la densité en fonction de la température de l'émetteur pour des conditions expérimentales identiques ( $V_{\mathrm{CE}}=-4$ volts, pression de césium $=10^{-2}$ torr $)$ sauf en ce qui concerne la distance émetteur collecteur $d$ qui a pour valeur $43 \mathrm{~mm}$ dans le cas des courbes (1) et (2) et $31 \mathrm{~mm}$ dans la cas des courbes (3) et (4).

La sonde axiale et la sonde radiale placées successivement au même point $(8 \mathrm{~mm}$ de l'émetteur, SA 8 et SRC) donnent les mêmes résultats pour des condi- 


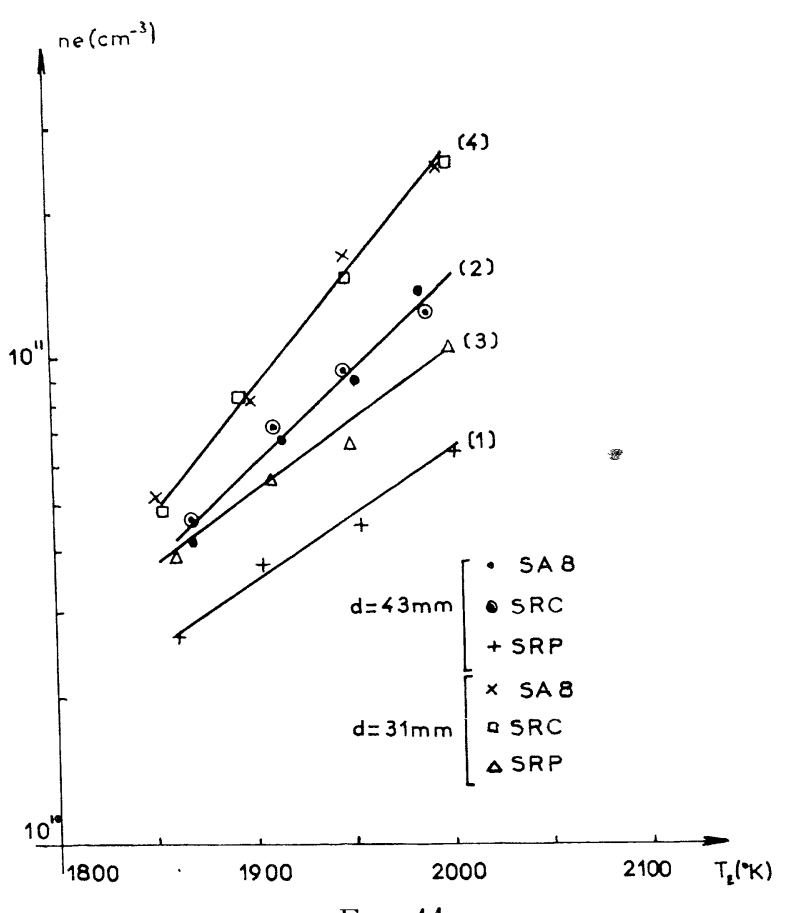

FIG. 11.

tions expérimentales identiques, comme on peut le voir sur les courbes (2) et (4).

On remarque en comparant ces courbes (2) et (4), où seule la distance émetteur collecteur varie, que la

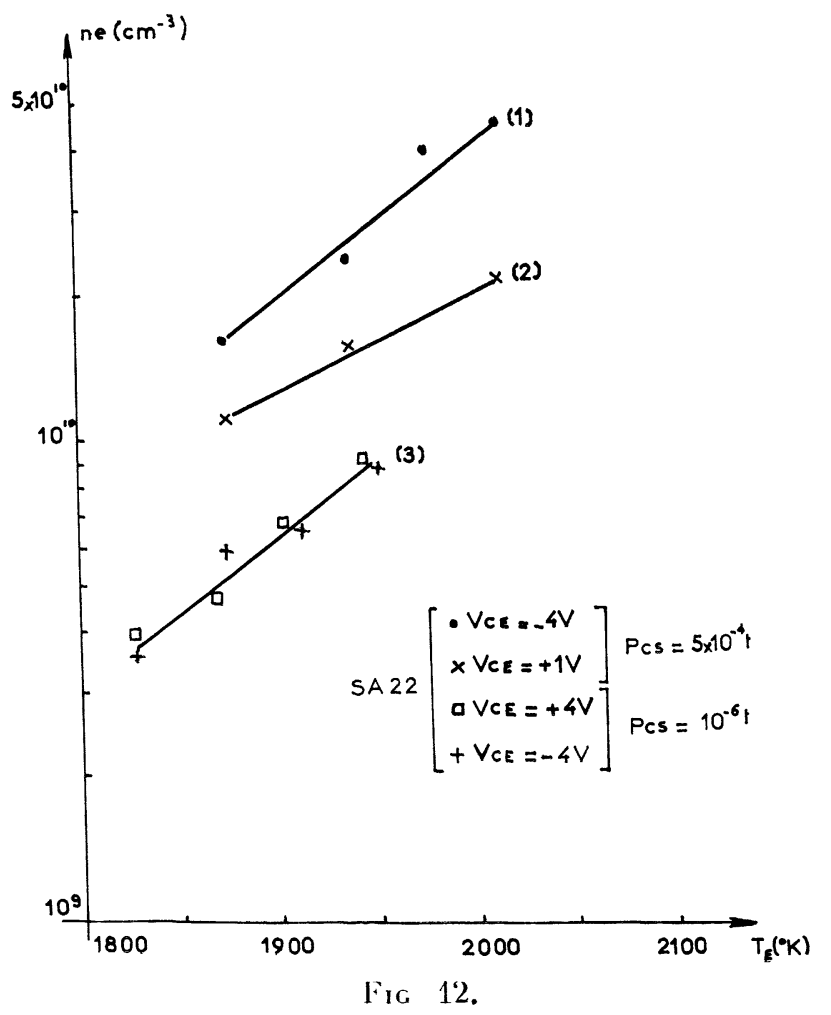

densité croît quand $d$ décrô̂t. Cet accroissement de densité est variable en fonction de la température de l'émetteur.

Les courbes 1 et 3 représentent les variations de densité obtenues dans les mêmes conditions que précédemment mais avec la sonde radiale placée en position périphérique. La pente de ces courbes est sensiblement plus faible en fonction de la température ainsi qu'en fonction de la distance émetteurcollecteur. Cette observation est normale puisque les mesures sont faites loin de l'axe.

Il semble que la distance émetteur-collecteur soit un des paramètres les plus importants mais elle est étroitement liée, quant à ses effets sur la densité, à la différence de potentiel imposée entre ces deux électrodes. L'influence de ces deux paramètres n'est pas simple, compte tenu des effets de paroi.

Etude de l'influence de la différence de potentiel du collecteur par rapport à l'émetteur. - Pour une pression de césium donnée et pour une température d'émetteur assurant une ionisation donnée, on peut théoriquement piéger les électrons dans un puits de potentiel en imposant une différence de potentiel collecteur-émetteur suffisamment négative.

Pour nous trouver dans ces conditions, nous avons choisi $V_{\mathrm{CE}}=-4$ volts. Néanmoins, nous avons modifié cette différence de potentiel et nous avons observé que la densité électronique mesurée en sonde axiale décroît quand $V_{\mathrm{CE}}$ croît. Ce fait est visible sur la figure 13 qui correspond aux conditions suivantes : la position de la sonde axiale est à $22 \mathrm{~mm}$ (SA 22) et la distance interélectrodes a pour valeur $d=43 \mathrm{~mm}$. Les courbes représentent les variations de densité électronique en fonction de la température de l'émetteur. Pour une pression de césium égale à $5 \times 10^{-4}$ torr, on a la courbe 1 qui correspond à $V_{\mathrm{CE}}=-4 \mathrm{~V}$ et la courbe 2 à $V_{\mathrm{CE}}=+1 \mathrm{~V}$ (on ne peut aller au delà sans provoquer des décharges). Les variations de densité sont aussi fonction de la pression de césium comme on peut le voir sur la courbe 3 qui est obtenue dans les mêmes conditions expérimentales que précédemment sauf en ce qui concerne la pression de césium qui est de $10^{-6}$ torr. Dans ce cas, la densité mesurée ne semble pas dépendre de $V_{\text {CE }}$ puisque les deux courbes expérimentales correspondant à $V_{\mathrm{CE}}=-4 \mathrm{~V}$ et à $V_{\text {CE }}=+4 \mathrm{~V}$ sont pratiquement confondues.

Les mêmes expériences ont été faites en sonde radiale et elles semblent montrer que la densité crô̂t plus vite en fonction de $T_{\mathbf{E}}$ quand $V_{\text {CE }}$ devient positive mais elles demandent confirmation.

Le fait que la mesure de densité dépende de la différence de potentiel collecteur émetteur peut être en rapport avec le mouvement des particules ionisées dans l'espace interélectrodes, lequel est étroitement lié aux configurations de potentiel.

En particulier quand $V_{\text {CE }}$ devient positive, il existe un important courant électronique entre 
l'émetteur et le collecteur et c'est ce qui pourrait expliquer la décroissance de densité observée. Remarquons encore que, dans ce cas, il y a diminution de pente de la courbe (2) par rapport à la courbe (1) ce qui n'était pas le cas quand seule la pression de césium variait.

Mesure du potentiel d'inflexion par les sondes mobiles. - Nous nous plaçons à une pression de césium bien définie $\left(10^{-4}\right.$ torr $)$ à une distance émetteur-collecteur fixe $(d=43 \mathrm{~mm})$ pour une différence de potentiel collecteur émetteur égale à - $4 \mathrm{~V}$.

On peut définir le potentiel dans un milieu ionisé à partir de la caractéristique de sonde en repérant la position du coude de la caractéristique tracée en coordonnées semi-logarithmiques $[25,27]$ ou en en considérant le point où la dérivée seconde $\mathrm{d}^{2} I / \mathrm{d} V^{2}$ s'annule $[25,29]$. Les deux méthodes ont été utilisées, elles donnent des résultats très voisins. Nous examinons comment varie le potentiel du point d'inflexion de la caractéristique en fonction de la température de l'émetteur pour différentes positions des sondes. Les résultats sont connus avec une précision moyenne de l'ordre de $0,2 \mathrm{~V}$. (L'erreur peut atteindre 0,5 volts dans certains cas défavorables.)

Sur la figure 13, la courbe 1 représente ces variations pour la sonde radiale en position périphérique.

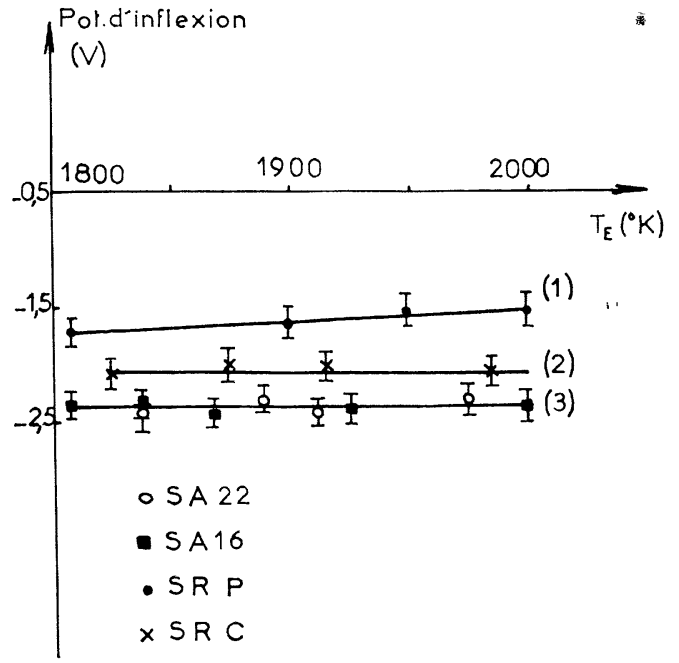

FIG. 13.

On constate que le potentiel d'inflexion varie légèrement quand la température d'émetteur croît. Ceci peut être dû à une modification du recouvrement en césium de l'électrode collectrice. La courbe 2 représente les variations du potentiel d'inflexion $V_{\mathbf{i}}$ pour la sonde radiale en position centrale (SRC). Dans ce cas $V_{\mathbf{i}}$ est sensiblement constant et il est légèrement plus négatif que précédemment. La courbe 3 représente, dans les mêmes conditions physiques les mesures du potentiel d'inflexion en sonde axiale pour deux positions. On observe que $V_{i}$ est sensiblement constant et qu'il ne semble pas dépendre de la position de la sonde sur l'axe. Le potentiel mesuré est inférieur aux précédents ce qui peut être dû à une variation du recouvrement. Il semble que le potentiel d'inflexion décroisse légèrement quand la pression de césium croît. Les indications de la sonde axiale ne semblent pas dépendre beaucoup de la tension collecteur-émetteur, tant qu'elle reste négative, si on n'est pas en régime d'arc. En ce qui concerne l'influence de la distance émetteur collecteur $d$, le potentiel d'inflexion de la sonde axiale ne semble pas en dépendre dans de larges limites. Les indications de la sonde radiale tendent moins à dépendre de sa position quand $d$ décroît. On peut dire que compte tenu de la précision de nos expériences, le potentiel d'inflexion mesuré par la sonde axiale semble surtout dépendre de la pression de césium.

En effet, l'isotropie du milieu n'est pas vérifiée avec nos deux sondes et on peut supposer qu'elles sont plongées dans un flux de particules. Dans ce cas le potentiel mesuré par notre méthode n'est pas celui du milieu mais il correspond à la différence des travaux de sortie de l'émetteur et de la sonde, ce qui expliquerait que la valeur mesurée soit constante. On observe un bon accord numérique avec les valeurs des travaux de sortie du tantale et du césium.

Mesure des températures électroniques. - Nous avons mesuré les températures électroniques, dans différentes conditions, sur la sonde axiale et sur la sonde radiale. La première conclusion que l'on peut tirer de ces mesures est que, dans des conditions de fonctionnement normal de la machine (c'est-à-dire quand la tension collecteur émetteur ne permet pas un régime d'arc), la distribution des vitesses obtenue à partir des caractéristiques des deux sondes, placées en différentes positions, est maxwellienne, comme on peut le voir sur les courbes tracées en coordonnées semi-logarithmiques. Par contre, en régime d'arc, nous trouvons deux distributions de vitesses. La deuxième conclusion concerne la mesure des températures électroniques : celles-ci sont obtenues avec une grande imprécision car de faibles erreurs (qui peuvent être dues à des indéterminations) dans l'exploitation des courbes caractéristiques de sonde se traduisent par de grands écarts numériques. (Plusieurs centaines de degrés, aussi les résultats sont-ils souvent connus à $\pm 25 \%$ près et quelquefois la précision est beaucoup plus faible.) Dans ces conditions, il est difficile de faire des mesures comparatives et nous pouvons principalement dire que les températures mesurées sont comprises entre $2000^{\circ}$ et $5000^{\circ} \mathrm{K}$.

Cependant malgré ces difficultés, nous avons représenté les points correspondants à la mesure de 
température électronique en fonction de la température de l'émetteur en les affectant de barres d'erreur qui correspondent à des imprécisions de l'ordre de 500 à 800 oK (quelquefois elles peuvent être supérieures). Les conditions expérimentales sont les suivantes : $d=43 \mathrm{~mm}, V_{\mathbf{C E}}=-4 \mathrm{~V}$, $P_{\text {cs }} \simeq \check{\jmath} \times 10^{-4}$ torr. Sur la figure 14, les points

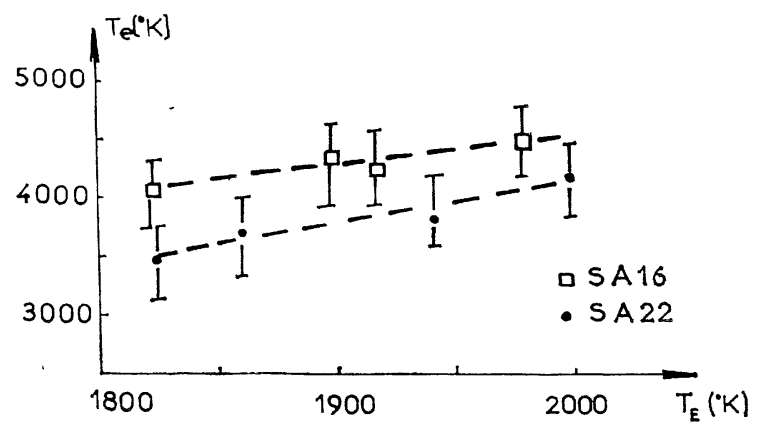

FIG. 14.

ont été obtenus avec la sonde axiale placée dans deux positions différentes (SA 16 et SA 22).

De même sur la figure 15 , nous avons représenté les points correspondants à la sonde radiale placée au

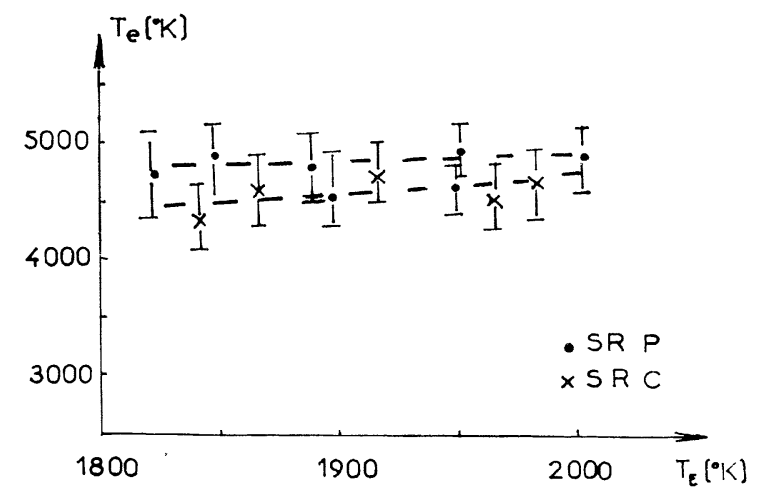

FIG. 15.

centre (SRC) et à la périphérie (SRP). Nous pouvons observer une certaine tendance variationnelle (courbes pointillées) mais, compte tenu des erreurs, il est impossible de conclure.

Notons seulement que les mesures obtenues pour nos températures électroniques semblent en bon accord avec d'autres travaux ([30], p. 180 et 181) et que, dans nos conditions de fonctionnement, la répartition des vitesses semble maxwellienne. Dans d'autres conditions expérimentales (en régime d'arc et à pression de césium de $10^{-2}$ torr) nous obtenons des électrons dont les températures sont plus élevées, alors que les distributions de vitesses ne sont plus maxwelliennes. Ils se répartissent en deux groupes d'énergie. Dans le premier cas, les électrons ont des températures comprises entre 6000 et $8000^{\circ} \mathrm{K}$ et dans le second, ces températures peuvent être supém rieures à $10000 \mathrm{~K}$.

Pour pouvoir comparer de manière plus précise les résultats fournis par la sonde axiale et la sonde radiale en fonction de leur position respective, il faut améliorer la précision des mesures et peut-être utiliser d'autres types de sondes.

Étude des variations de densité en régime d'arc. - Pour une différence de potentiel collecteur émetteur suffisamment positive (elle dépend de la pression de césium) il s'établit un arc entre les électrodes. Le phénomène est lumineux et le courant traversant la machine peut être élevé. La figure 16 représente dans les mêmes conditions physiques (pression de césium $10^{-3}$ torr, $d=43 \mathrm{~mm}$ ) des mesures

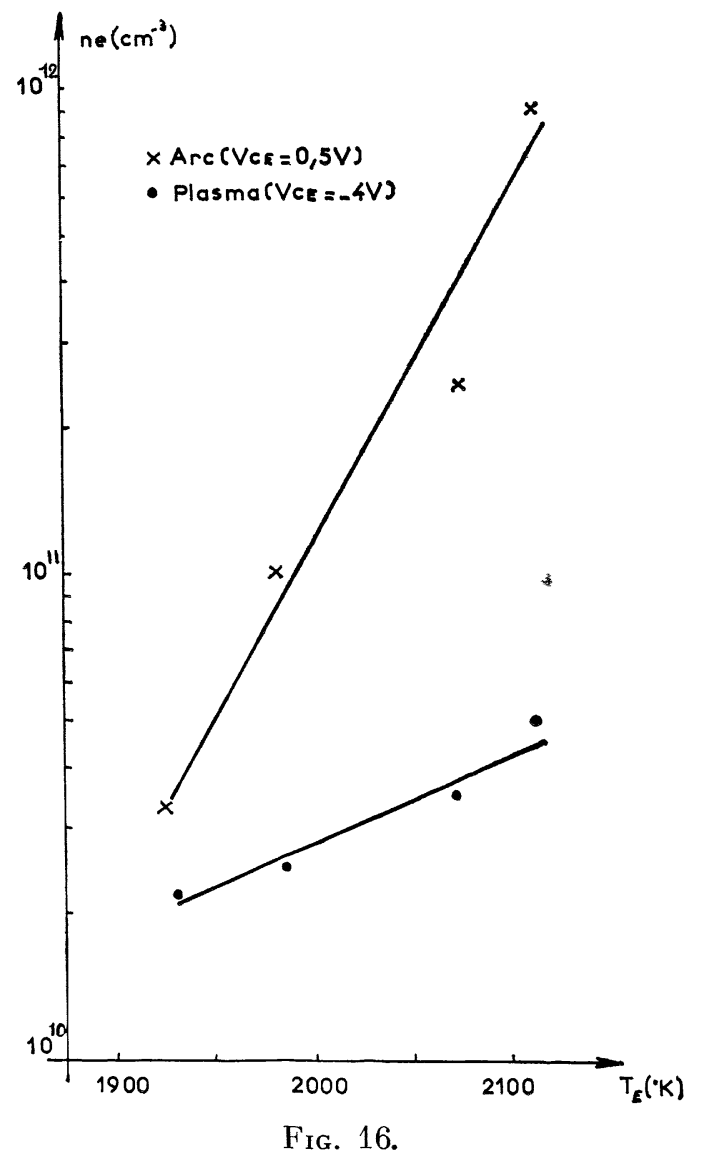

de densité en régime normal $\left(V_{\mathrm{CE}}=-4 \mathrm{~V}\right)$ et en régime d'arc $V_{\mathrm{CE}}=+0,5 \mathrm{~V}$ ) avec la sonde radiale en position périphérique (SRP) car elle présente les caractéristiques les plus facilement exploitables. Nous remarquons que la densité en régime d'arc croît très rapidement et elle peut atteindre $10^{12}$ électrons par centimètre cube pour une température d'émetteur de l'ordre de $2100^{\circ} \mathrm{K}$.

Ces résultats sont en accord avec d'autres travaux [31] et nous trouvons que la température des 
électrons n'est plus maxwellienne. La caractéristique de sonde tracée en coordonnées semi-logarithmiques présente deux segments de droite et il semble que l'on se trouve en présence de deux groupes d'électrons d'énergie différente.

Mesure en sondes à résonance. - Cette technique est bien connue [32,33] mais dans notre cas son utilisation est limitée, pour des raisons matérielles, par la fréquence de résonance qui ne doit pas être trop élevée pour que nous puissions faire la mesure. Nous nous sommes donc proposé de faire quelques mesures pour des faibles densités, celles-ci étant principalement destinées à confirmer les résultats acquis au moyen des sondes de Langmuir.

Par exemple (fig. 17) pour $T_{\mathrm{E}}=1450^{\circ} \mathrm{K}$, $P_{\mathrm{cs}}=10^{-6}$ torr et $V_{\mathrm{CE}}=-4 \mathrm{~V}$ la fréquence

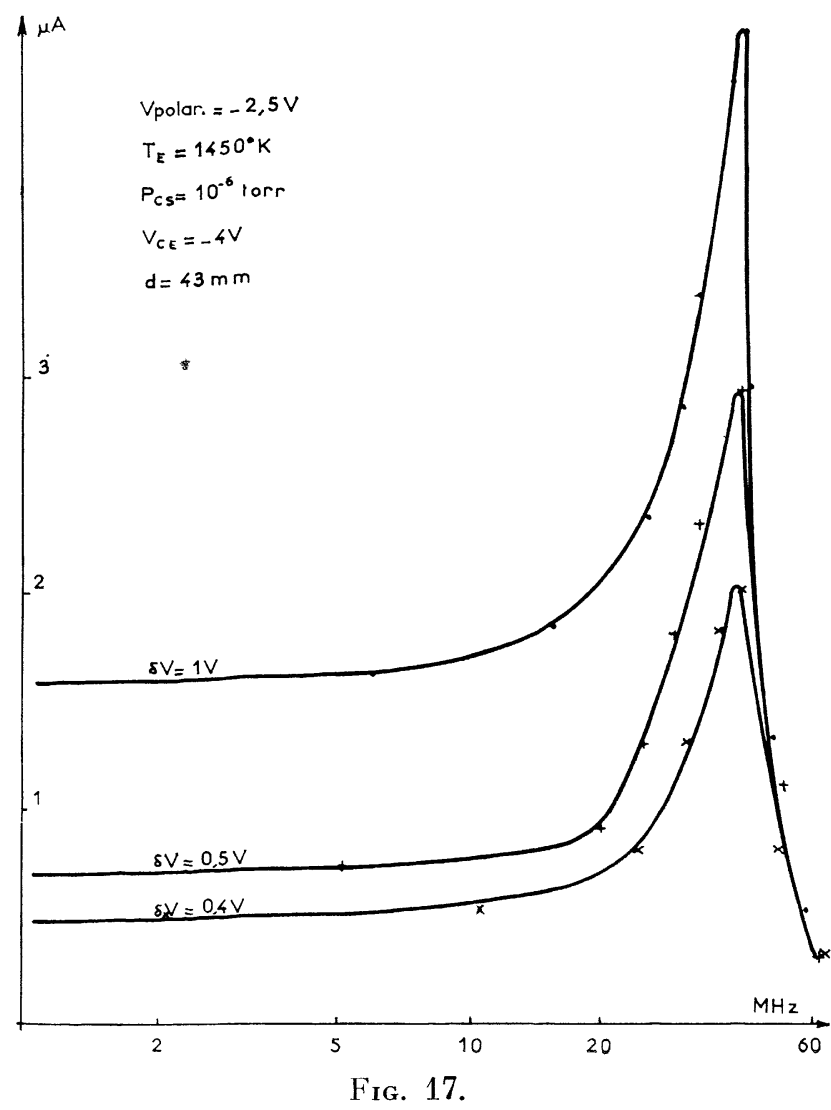

d'antirésonance est égale à $60 \mathrm{MHz}$ ce qui donne une densité de $1,2 \times 10^{7} \mathrm{e} / \mathrm{cm}^{3}$; une température d'électrons de $1500^{\circ} \mathrm{K}$ et une fréquence de collision électrons-neutres de $5 \times 10^{6}$ collisions par seconde. Dans les mêmes conditions une sonde de Langmuir donne une densité électronique voisine de $4 \times 10^{7} \mathrm{e} / \mathrm{cm}^{3}$. On peut donc considérer ces résultats comme satisfaisants.

Conclusion. - Nous avons réalisé un certain nombre de mesures avec notre machine qui était soumise à des conditions expérimentales variées. Nous avons essayé de déterminer l'influence respective des différents paramètres sur son régime de fonctionnement mais l'exploitation des résultats est délicate pour deux raisons importantes :

La première provient du fait que les relations mutuelles entre ces différents paramètres ne sont pas simples, quand elles sont connues, ce qui n'est pas toujours le cas.

La deuxième raison concerne la mauvaise reproductibilité des résultats, conséquence entre autres choses de la mauvaise estimation de la pression de césium dans la machine. En effet, nous ne disposons pas de technique nous donnant directement cette pression et la méthode utilisée peut conduire à de nombreuses erreurs. De plus ce corps réagit fortement avec la plupart des autres éléments et il donne lieu à des phénomènes de surface complexes et mal connus. Quand la machine est remise au contact de l'air il y a formation d'oxydes qui ne sont pas facilement éliminés. Il existe aussi d'autres sources d'erreurs importantes telles que la mesure des températures de la surface chaude et de l'extrémité des sondes ou l'évaluation de la surface de collection des sondes. Certaines parties de l'enceinte ont des températures élevées par suite de la présence de l'émetteur qui rayonne de l'énergie ; ces surfaces recouvertes de césium ont une émission qui peut devenir importante. Enfin, la géométrie joue, elle aussi, un rôle important et l'influence des parois n'est plus négligeable quand la distance collecteur-émetteur est grande.

Compte tenu de ces imprécisions qui rendent difficile toute mesure absolue, on peut cependant faire des études comparatives quand les conditions de fonctionnement de la machine sont fixées au cours d'un cycle d'expériences. C'est ce que nous nous sommes proposés de faire.

La pression de césium et le libre parcours moyen des électrons semblent être les facteurs déterminants, par exemple dans les mêmes conditions expérimentales et pour $T_{\mathbf{E}}=1950^{\circ} \mathrm{K}$, on observe que quand la pression de césium est multipliée par un facteur $20\left(5 \times 10^{-4}\right.$ à $10^{-2}$ torr $)$ la densité électronique mesurée par la sonde axiale, placée sensiblement au centre, est multipliée par un facteur moyen voisin de 2,3 alors que cette même densité mesurée par la sonde radiale (proche de l'émetteur) placée au voisinage de l'axe (3 $\mathrm{mm}$ environ) est multipliée par un facteur moyen voisin de 2,6 . Dans les mêmes conditions le facteur multiplicatif moyen est voisin de 3,5 pour la sonde radiale en position périphérique, ceci montre que la densité électronique mesurée dans les mêmes conditions physiques dépend de la position des sondes, ce qui est naturel puisque le milieu n'est pas homogène. Quand $d$ varie de $30 \%$, les autres paramètres restant constants, la densité est multipliée par un facteur voisin de 1,7 pour la sonde radiale, qu'elle soit en position 
périphérique ou proche de l'axe (évaluation moyenne faite pour $\left.T_{\mathrm{E}}=1950 \mathrm{~K}\right)$. En conclusion, on peut dire que, dans nos expériences, les renseignements obtenus sur le milieu ionisé à partir des mesures de sondes dépendent de la position de celles-ci. Elles sont en accord avec d'autres résultats [34]. Cette constatation est naturelle puisque le milieu n'est pas homogène dans notre machine compte tenu de sa géométrie et en particulier de la grande distance interélectrodes pour laquelle les parois peuvent avoir de l'influence. Un de nos objectifs est de pouvoir obtenir des informations plus précises concernant la distribution du potentiel, de la densité et de la température électronique, ces renseignements étant nécessaires avant toute interprétation théorique. On peut conclure qu'il faut tenir compte des méthodes de mesure et de la manière dont celles-ci sont faites, dans toute tentative d'interprétation. Notre machine permet d'étudier certains problèmes concernant les processus d'échange d'énergie, d'ionisation de contact, d'oscillations [35], de recombinaison. Si de nombreux travaux ont déjà été menés sur ces sujets, les expériences ne sont pas encore nombreuses et l'étude du césium ionisé fournit de nombreux renseignements intéressants. En effet, il permet d'obtenir un plasma stable dont les propriétés sont différentes de celles qui caractérisent les plasmas obtenus en décharge

Remerciements. - Les auteurs remercient le Conseil Général de Meurthe-et-Moselle et la Ville de Nancy qui ont entièrement financé ces recherches.

\section{BIBLIOGRAPHIE}

[1] Langmuir et Kingdom, Phys. Res., 1923, 21, 380 ; Proc. Roy. Soc., 1925, A 107, 61.

[2] ZANDBERG et Ionov, Uspecki, 1959, 57, vol. 67, $n^{\circ} 2,581$.

[3] Le Binan et Maugis, Annales de Radioélectricité, 1965, 20, no 80.

[4] Richardon, Proc. Cam. Phil., 1902, 11, 286.

[5] Blanc-Lapierre, Goudet et Lapostolle, Électronique générale, Eyrolles, 1953.

[6] Notringham, Hand. Physik, Spinger Verlag, 1956, 21, 16.

[7] Defranould, Thèse, Paris, mai 1963.

[8] Van Leimpt, Recueil des travaux chimiques des Pays-Bas, 1936, 55-157.

[9] Houston et Webster, Advances in electronics, Academic Press, 1962.

[10] Delafosse et Mongodin, Calculs de la technique $\mathrm{d} u$ vide. Soc. Française Ing. Tech. du vide.

[11] Langmuir et Taylor, Phys. Rev., 1933, 44, no 6.

[12] Notringham, Conf. on ionisation ph. in gazes, Uppsala, Suède, a oût 1959, p. 486-501.

[13] Nottingham, Direct conversion on heat to electricity, Wiley, 1960.

[14] Medicus, J. Appl. Physics, 1962, 33, no 10, 3094.

[15] Notringham, S. Flügge, Springer Verlag, 1961, XXI.

[16] Houston, Bull. Amer. Phys. Soc., (2) 1961, 6, 358.

[17] Brode, Phys. Rev., 1929, 34, 673.

[18] Brode, Rev. Mod. Physics, 1933, 5, 257.

[19] Chapman, Caulfield et Hemstreet, J. Appl. Physics, 1964, 35, no 10, 2813.

[20] Yang, Ling et Carpenter, Electrochem. Soc. Jap., novembre 1961, 1079-1804.
[21] Smith et Hargreaves, J. nuclear materials, 1963, 191-200.

[22] Dushmann, Scientific foundations of vacuum"techniques, Wiley, 1962.

[23] Rosebury, Handbook of electron tubes and vacuum techniques, Addison Wesley, 1965.

[24] Kонг, Materials and techniques for electrons tubes, Reinhold Publi. Corp., New York, 1960.

[25] Loев, Basic processes of gazeous electronics, Univ. of Calif., 1961.

[26] Bullis et Wiegand, United aircraft corporation 23th conf. on physica electronics, M. I. T., 22 mars 1963.

[27] Fitaire, Rapport LP. 45, mai 1965.

[28] Felden, Haug et Patou, C. R. Acad. Sc., 1965, 261, 1215-1218.

[29] Rapport interne L. P. T. N., Les techniques d'enregistrement de caractéristiques de sondes et de leurs dérivées.

[30] Houston et Webster, Advance in electronics and electrons physics, Academic Press.

[31] Bullis et Wiegand, U. S. Dept. of Com. Report AD 411.474.

[32] Ichinawa et Inegami, Prob. Theor. Physics, 1962, 28, 311.

[33] Peter, Muller et Rabben, IV Int. Conf., Paris, 1963.

[34] Felden, Patou, Haug et Schirmann, $C . R$. Acad. Sc., 1965, 261, 3321-3324.

[35] Felden,"Haug et Patou, C. R. Acad. Sc., 1965, 261, 1480-1483. 